\title{
TRACES OF PERMUTING GENERALIZED $N$-DERIVATIONS OF RINGS
}

\author{
MOHAMMAD ASHRAF, ALMAS KHAN, AND MALIK RASHID JAMAL
}

Received 03 November, 2015

\begin{abstract}
Let $n \geq 1$ be a fixed positive integer and $R$ be a ring. A permuting $n$-additive map $\Omega: R^{n} \rightarrow R$ is known to be permuting generalized $n$-derivation if there exists a permuting $n$ derivation $\Delta: R^{n} \rightarrow R$ such that $\Omega\left(x_{1}, x_{2}, \cdots, x_{i} x_{i}^{\prime}, \cdots, x_{n}\right)=\Omega\left(x_{1}, x_{2}, \cdots, x_{i}, \cdots, x_{n}\right) x_{i}^{\prime}+$ $x_{i} \Delta\left(x_{1}, x_{2}, \cdots, x_{i}^{\prime}, \cdots, x_{n}\right)$ holds for all $x_{i}, x_{i}^{\prime} \in R$. A mapping $\delta: R \rightarrow R$ defined by $\delta(x)=$ $\Delta(x, x, \cdots, x)$ for all $x \in R$ is said to be the trace of $\Delta$. The trace $\omega$ of $\Omega$ can be defined in the similar way. The main result of the present paper states that if $R$ is a $(n+1)$ !-torsion free semi-prime ring which admits a permuting $n$-derivation $\Delta$ such that the trace $\delta$ of $\Delta$ satisfies $[[\delta(x), x], x] \in Z(R)$ for all $x \in R$, then $\delta$ is commuting on $R$. Besides other related results it is also shown that in a $n$ !-torsion free prime ring if the trace $\omega$ of a permuting generalized $n$-derivation $\Omega$ is centralizing on $R$, then $\omega$ is commuting on $R$.
\end{abstract}

2010 Mathematics Subject Classification: 16W25; 16U80

Keywords: derivation, generalized derivation, permuting $n$-derivation, centralizing map, prime ring

\section{INTRODUCTION}

Throughout $R$ will denote an associative ring with center $Z(R)$. For any $x, y \in R$, $x y-y x$ denote the commutator $[x, y]$. A ring $R$ is said to be prime (resp. semiprime) if $a R b=\{0\}$ implies either $a=0$ or $b=0$ (resp. $a R a=\{0\}$ implies $a=0$ ). Let $m \geq 1$ be a fixed positive integer. A map $f: R \rightarrow R$ is said to be centralizing (resp. commuting) on $R$ if $[f(x), x] \in Z(R)$ (resp. $[f(x), x]=0$ ) holds for all $x \in R$. An additive mapping $d: R \rightarrow R$ is called a derivation if $d(x y)=d(x) y+x d(y)$ holds for all $x, y \in R$. Following [4], an additive mapping $F: R \longrightarrow R$ is said to be a generalized derivation on $R$ if there exists a derivation $d: R \longrightarrow R$ such that $F(x y)=F(x) y+x d(y)$ holds for all $x, y \in R$. Suppose $n$ is a fixed positive integer and $R^{n}=R \times R \times \cdots \times R$. A map $\Delta: R^{n} \rightarrow R$ is said to be permuting if the relation $\Delta\left(x_{1}, x_{2}, \cdots, x_{n}\right)=\Delta\left(x_{\pi(1)}, x_{\pi(2)}, \cdots, x_{\pi(n)}\right)$ holds for all $x_{i} \in R$ and for every permutation $\{\pi(1), \pi(2), \cdots, \pi(n)\}$. The concept of derivation and symmetric bi-derivation was generalized by Park [7] as follows: a permuting map $\Delta: R^{n} \rightarrow R$ is said to be a permuting $n$-derivation if $\Delta$ is $n$-additive (i.e.; additive 
in each coordinate) and $\Delta\left(x_{1}, x_{2}, \cdots, x_{i} x_{i}^{\prime}, \cdots, x_{n}\right)=x_{i} \Delta\left(x_{1}, x_{2}, \cdots, x_{i}^{\prime}, \cdots, x_{n}\right)+$ $\Delta\left(x_{1}, x_{2}, \cdots, x_{i}, \cdots, x_{n}\right) x_{i}^{\prime}$ holds for all $x_{i}, x_{i}^{\prime} \in R$. A 1-derivation is a derivation and a 2-derivation is a symmetric bi-derivation while a 3-derivation is known as permuting tri-derivation.

A well known result due to Posner [8] states that a prime ring $R$ which admits a non-zero centralizing derivation is commutative. In fact, this result initiated the study of centralizing and commuting mappings in rings. Since then, several authors have done a great deal of work concerning commutativity of prime and semi-prime rings admitting different kinds of maps which are centralizing or commuting on some appropriate subsets of $R$ (see [5,6] and [7] for further references). Let $n \geq 2$ be a fixed integer and a map $\delta: R \rightarrow R$ defined by $\delta(x)=\Delta(x, x, \cdots, x)$ for all $x \in R$, where $\Delta: R^{n} \rightarrow R$ is a permuting map, be the trace of $\Delta$. Moreover, it can be easily seen that $\Delta\left(x_{1}, x_{2}, \cdots,-x_{i}, \cdots, x_{n}\right)=-\Delta\left(x_{1}, x_{2}, \cdots, x_{i}, \cdots, x_{n}\right)$ for all $x_{i} \in R, i=1,2, \cdots, n$.

Motivated by the concept of generalized derivation in ring, we introduce the notion of permuting generalized $n$-derivation in ring. Let $n \geq 1$ be a fixed positive integer. A permuting $n$-additive map $\Omega: R^{n} \rightarrow R$ is known to be permuting generalized $n$-derivation if there exists a permuting $n$-derivation $\Delta: R^{n} \rightarrow R$ such that $\Omega\left(x_{1}, x_{2}, \cdots, x_{i} x_{i}^{\prime}, \cdots, x_{n}\right)=\Omega\left(x_{1}, x_{2}, \cdots, x_{i}, \cdots, x_{n}\right) x_{i}^{\prime}+x_{i} \Delta\left(x_{1}, x_{2}\right.$, $\left.\cdots, x_{i}^{\prime}, \cdots, x_{n}\right)$ holds for all $x_{i}, x_{i}^{\prime} \in R$. For an example of permuting generalized $n$-derivation, let $n \geq 1$ be a fixed positive integer and $R=\left\{\left(\begin{array}{ccc}0 & a & b \\ 0 & 0 & c \\ 0 & 0 & 0\end{array}\right) \mid a, b, c \in\right.$ $\mathbb{C}\}$ where $\mathbb{C}$ is a complex field. Consider permuting $n$-derivation $\Delta$ as above and define $\Omega: R^{n} \rightarrow R$ such that

$$
\Omega\left(\left(\begin{array}{ccc}
0 & a_{1} & b_{1} \\
0 & 0 & c_{1} \\
0 & 0 & 0
\end{array}\right), \cdots,\left(\begin{array}{ccc}
0 & a_{n} & b_{n} \\
0 & 0 & c_{n} \\
0 & 0 & 0
\end{array}\right)\right)=\left(\begin{array}{ccc}
0 & 0 & a_{1} \cdots a_{n} \\
0 & 0 & c_{1} \cdots c_{n} \\
0 & 0 & 0
\end{array}\right) .
$$

Then $\Omega$ is a permuting generalized $n$-derivation on $R$ associated with a permuting $n$-derivation $\Delta$ on $R$.

Let $\omega: R \rightarrow R$ such that $\omega(x)=\Omega(x, x, \cdots, x)$. Then $\omega$ is known as the trace of $\Omega$. A permuting $n$-additive map $\Lambda: R^{n} \rightarrow R$ is said to be a permuting left $n$-multiplier (resp. permuting right $n$-multiplier) if $\Lambda\left(x_{1}, x_{2}, \cdots, x_{i} x_{i}^{\prime}, \cdots, x_{n}\right)=$ $\Lambda\left(x_{1}, x_{2}, \cdots, x_{i}, \cdots, x_{n}\right) x_{i}^{\prime}$ (resp. $\quad \Lambda\left(x_{1}, x_{2}, \cdots, x_{i} x_{i}^{\prime}, \cdots, x_{n}\right)=x_{i} \Lambda\left(x_{1}, x_{2}, \cdots\right.$, $\left.x_{i}^{\prime}, \cdots, x_{n}\right)$ ) holds for all $x_{i}, x_{i}^{\prime} \in R$. If $\Lambda$ is both permuting left $n$-multiplier as well as right $n$-multiplier, then $\Lambda$ is called a permuting $n$-multiplier.

Motivated by the results due to Posner [8], Vukman obtained some results concerning the trace of symmetric bi-derivation in prime ring (see [9, 10]). Ashraf [1] proved similar results for semi-prime ring. In the year 2009, Park [7] introduced the concept of symmetric permuting $n$-derivation and obtained some results related to 
the commuting traces of permuting $n$-derivations in rings. Further, the first author together with Jamal and Parveen [2,3] obtained commutativity of rings admitting $n$-derivations whose traces satisfy certain polynomial conditions.

The main objective of this paper is to find the analogous results for permuting generalized $n$-derivation in the setting of prime and semi-prime rings. In fact, our theorems present a wide generalization of the results obtained in [1], Theorem 2.1, [7], Theorem 2.3, [7], Theorem 2.5, [9], Theorem 1, [9], Theorem 2, [10], Theorem 2 etc.

\section{Results}

We begin with the following known results which are frequently used in our discussion.

Lemma 1 (Lemma 2.4 in [7]). Let $n$ be a fixed positive integer and let $R$ be a $n$ !torsion free ring. Suppose that $y_{1}, y_{2}, \cdots, y_{n} \in R$ satisfy $\lambda y_{1}+\lambda^{2} y_{2}+\cdots+\lambda^{n} y_{n}=$ $0($ or $\in Z(R))$ for $\lambda=1,2, \cdots, n$. Then $y_{i}=0\left(\right.$ or $\left.y_{i} \in Z(R)\right)$ for all $i$.

Lemma 2 (Theorem 2.3 in [7]). Let $n \geq 2$ be a fixed positive integer and $R$ be $a$ non-commutative $n$ !-torsion free prime ring. Suppose that there exists a permuting $n$-derivation $\Delta: R^{n} \rightarrow R$ such that the trace $\delta$ of $\Delta$ is commuting on $R$. Then we have $\Delta=0$

Lemma 3 (Theorem 2.6 in [7]). Let $n \geq 2$ be a fixed positive integer and $R$ be a $n$ !torsion free prime ring. Suppose that there exists a non-zero permuting $n$-derivation $\Delta: R^{n} \rightarrow R$ such that the trace $\delta$ of $\Delta$ is centralizing on $R$ then $R$ is commutative.

As stated in the beginning, there has been a great deal of work concerning centralizing and commuting mappings. The following result shows that if the trace $\delta$ of a permuting $n$-derivation $\Delta$ is centralizing on $R$ then it is commuting on $R$. In fact, we prove rather a more general result:

Theorem 1. Let $n \geq 2$ be a fixed positive integer and $R$ be a $(n+1)$ !-torsion free semi-prime ring admitting a permuting $n$-derivation $\Delta$ such that the trace $\delta$ of $\Delta$ satisfies $[[\delta(x), x], x]=0$ for all $x \in R$. Then $\delta$ is commuting on $R$.

Proof. From our hypothesis we have

$$
[[\delta(x), x], x]=0 \text { for all } x \in R .
$$

An easy computation shows that the traces $\delta$ of $\Delta$ satisfies the following relations

$$
\delta(x+y)=\delta(x)+\delta(y)+\sum_{r=1}^{n-1}\left(\begin{array}{l}
n \\
r
\end{array}\right) h_{r}(x, y) \text { for all } x, y \in R
$$

where $h_{r}(x, y)=\Delta(\underbrace{x, x, \cdots, x}_{(n-r)-\text { times }}, \underbrace{y, y, \cdots, y}_{r-\text { times }})$. 
Consider a positive integer $k, 1 \leq k \leq n+1$. Replacing $x$ by $x+k y$ in equation (2.1), we obtain

$$
k Q_{1}(x, y)+k^{2} Q_{2}(x, y)+\ldots+k^{n+1} Q_{n+1}(x, y)=0 \text { for all } x, y \in R,
$$

where $Q_{i}(x, y)$ denotes the sum of the terms in which $y$ appears $i$ times. By (2.1) and Lemma 1, we have for all $x, y \in R$,

$$
[[\delta(x), x], y]+[[\delta(x), y], x]+n[[\Delta(x, x, \ldots, y), x], x]=0 .
$$

Replacing $y$ by $x y$ in (2.2) we get

$$
\begin{aligned}
0= & {[[\delta(x), x], x y]+[[\delta(x), x y], x]+n[[\Delta(x, x, \ldots, x y), x], x] } \\
= & {[[\delta(x), x], x y]+[[\delta(x), x y], x]+n[[x \Delta(x, x, \ldots, y), x], x] } \\
& +n[[\delta(x) y, x], x] \\
= & {[[\delta(x), x], x y]+[x[\delta(x), y], x]+[[\delta(x), x] y, x] } \\
& +n[x[\Delta(x, x, \ldots, y), x], x]+n[\delta(x)[y, x], x]+n[[\delta(x), x] y, x] \\
= & {[[\delta(x), x], x] y+x[[\delta(x), x], y]+x[[\delta(x), y], x]+[x, x][\delta(x), y] } \\
& +[\delta(x), x][y, x]+[[\delta(x), x], x] y+n x[[\Delta(x, x, \ldots, y), x], x] \\
& +n \delta(x)[[y, x], x]+n[\delta(x), x][y, x]+n[\delta(x), x][y, x]+n[[\delta(x), x], x] y .
\end{aligned}
$$

Using (2.1) and (2.2) we find that

$$
(2 n+1)[\delta(x), x][y, x]+n \delta(x)[[y, x], x]=0 \text { for all } x, y \in R .
$$

Similarly, replacing $y$ by $y x$ in (2.2), one can get

$$
(2 n+1)[y, x][\delta(x), x]+n[[y, x], x] \delta(x)=0 \text { for all } x, y \in R .
$$

Replacing $y$ by $y z$ in (2.3), we have

$$
\begin{aligned}
0= & (2 n+1)[\delta(x), x][y z, x]+n \delta(x)[[y z, x], x] \\
= & (2 n+1)\{[\delta(x), x][y, x] z+[\delta(x), x] y[z, x]\}+n \delta(x) y[[z, x], x] \\
& +n \delta(x)[y, x][z, x]+n \delta(x)[y, x][z, x]+n \delta(x)[[y, x], x] z .
\end{aligned}
$$

Using equation (2.3)

$$
(2 n+1)[\delta(x), x] y[z, x]+n \delta(x) y[[z, x], x]+2 n \delta(x)[y, x][z, x]=0 .
$$

Replacing $y$ by $\delta(x)$ in the above relation we find that

$$
(2 n+1)[\delta(x), x] \delta(x)[z, x]+n \delta(x)^{2}[[z, x], x]+2 n \delta(x)[\delta(x), x][z, x]=0 .
$$

From (2.3) we have $n \delta(x)^{2}[[y, x], x]=-(2 n+1) \delta(x)[\delta(x), x][y, x]$. Now using this relation in (2.5) we get

$$
\begin{aligned}
0= & (2 n+1)[\delta(x), x] \delta(x)[z, x]-(2 n+1) \delta(x)[\delta(x), x][z, x] \\
& +2 n \delta(x)[\delta(x), x][z, x] \\
= & \{(2 n+1)[\delta(x), x] \delta(x)-\delta(x)[\delta(x), x]\}[z, x] .
\end{aligned}
$$


Similarly using (2.4) one can easily obtain

$$
\{(2 n+1) \delta(x)[\delta(x), x]-[\delta(x), x] \delta(x)\}[z, x]=0 .
$$

Adding (2.6) and (2.7) we arrive at

$$
2 n\{[\delta(x), x] \delta(x)+2 n \delta(x)[\delta(x), x]\}[z, x]=0 .
$$

Since $2 n$ divides $(n+1)$ !, we find that $R$ is $2 n$-torsion free and hence for all $x, z \in R$,

$$
\{[\delta(x), x] \delta(x)+\delta(x)[\delta(x), x]\}[z, x]=0 .
$$

Using (2.8) in (2.6) we obtain $(2 n+2)[\delta(x), x] \delta(x)[z, x]=0$ for all $x, z \in R$. Since $2(n+1)$ divides $(n+1)$ !, we find that $R$ is $2(n+1)$-torsion free and hence for all $x, z \in R$,

$$
[\delta(x), x] \delta(x)[z, x]=0 \text { for all } x, z \in R \text {. }
$$

Substituting $y z$ for $z$ we get $[\delta(x), x] \delta(x) y[z, x]=0$ for all $x, y, z \in R$. Replacing $z$ by $\delta(x)$ we obtain $0=[\delta(x), x] \delta(x) y[\delta(x), x] \delta(x)$. Semiprimeness of $R$ yields

$$
[\delta(x), x] \delta(x)=0 \text { for all } x \in R .
$$

Similarly application of (2.7) and (2.8) yields that

$$
\delta(x)[\delta(x), x]=0 \text { for all } x \in R .
$$

Replacing $x$ by $x+k y$ in equation (2.10) where $1 \leq k \leq 2 n$ and implementing Lemma 1

$$
\delta(x)[\delta(x), y]+n \delta(x)[\Delta(x, x, \ldots, y), x]+n \Delta(x, x, \ldots, y)[\delta(x), x]=0 .
$$

Replacing $y$ by $y x$

$$
\begin{aligned}
0= & \delta(x)[\delta(x), y x]+n \delta(x)[y \delta(x)+\Delta(x, x, \ldots, y) x, x] \\
& +n\{y \delta(x)+\Delta(x, x, \ldots, y) x\}[\delta(x), x] \\
= & \delta(x) y[\delta(x), x]+\delta(x)[\delta(x), y] x+n \delta(x) y[\delta(x), x] \\
& +n \delta(x)[y, x] \delta(x)+n \delta(x)[\Delta(x, x, \ldots, y), x] x \\
& +n y \delta(x)[\delta(x), x]+n \Delta(x, x, \ldots, y) x[\delta(x), x] .
\end{aligned}
$$

From (2.11) we have

$$
-n \Delta(x, x, \ldots, y)[\delta(x), x] x=\delta(x)[\delta(x), y] x+n \delta(x)[\Delta(x, x, \ldots, y), x] x .
$$

Using (2.10) and (2.12) in the above relation, we get

$$
\begin{aligned}
0= & (n+1) \delta(x) y[\delta(x), x]+n \delta(x)[y, x] \delta(x)+n \Delta(x, x, \ldots, y) x[\delta(x), x] \\
& -n \Delta(x, x, \ldots, y)[\delta(x), x] x \\
= & (n+1) \delta(x) y[\delta(x), x]+n \delta(x)[y, x] \delta(x)-n \Delta(x, x, \ldots, y)[[\delta(x), x] x] .
\end{aligned}
$$

This gives that

$$
(n+1) \delta(x) y[\delta(x), x]+n \delta(x)[y, x] \delta(x)=0 \text { for all } x, y \in R .
$$


Substituting $x y$ for $y$ in (2.13)

$$
(n+1) \delta(x) x y[\delta(x), x]+n \delta(x) x[y, x] \delta(x)=0 \text { for all } x, y \in R .
$$

Left multiply (2.13) by $x$, we obtain

$$
(n+1) x \delta(x) y[\delta(x), x]+n x \delta(x)[y, x] \delta(x)=0 \text { for all } x, y \in R .
$$

Combining (2.14) and (2.15), we get

$$
(n+1)[\delta(x), x] y[\delta(x), x]+n[\delta(x), x][y, x] \delta(x)=0 \text { for all } x, y \in R .
$$

Replacing $y$ by $y z$ in (2.4), we obtain

$$
\begin{aligned}
0= & (2 n+1)[y z, x][\delta(x), x]+n[[y z, x], x] \delta(x) \\
= & (2 n+1)[y z, x][\delta(x), x]+n[y[z, x], x] \delta(x)+n[[y, x] z, x] \delta(x) \\
= & (2 n+1) y[z, x][\delta(x), x]+(2 n+1)[y, x] z[\delta(x), x]+n y[[z, x], x] \delta(x) \\
& +n[y, x][z, x] \delta(x)+n[y, x][z, x] \delta(x)+n[[y, x], x] z \delta(x) .
\end{aligned}
$$

Using (2.4) we get,

$$
(2 n+1)[y, x] z[\delta(x), x]+2 n[y, x][z, x] \delta(x)+n[[y, x], x] z \delta(x)=0 .
$$

Replacing $y$ by $\delta(x)$ in the above relation we get

$$
(2 n+1)[\delta(x), x] z[\delta(x), x]+2 n[\delta(x), x][z, x] \delta(x)=0 \text { for all } x, z \in R .
$$

Combining equations (2.16) and (2.17) we find that

$$
\begin{aligned}
0 & =(2 n+1)[\delta(x), x] z[\delta(x), x]-2(n+1)[\delta(x), x] z[\delta(x), x] \\
& =[\delta(x), x] z[\delta(x), x] \text { for all } x, z \in R .
\end{aligned}
$$

Since $R$ is semi-prime, we get $[\delta(x), x]=0$, for all $x \in R$.

Theorem 2. Let $n \geq 2$ be a fixed positive integer and $R$ be a $(n+1)$ !-torsion free semi-prime ring admitting a permuting $n$-derivation $\Delta$ such that the trace $\delta$ of $\Delta$ satisfies $[[\delta(x), x], x] \in Z(R)$ for all $x \in R$. Then $\delta$ is commuting on $R$.

Proof. Replace $x$ by $x+k y$ for $1 \leq k \leq n+1$ in the given condition to find that

$$
k Q_{1}(x, y)+k^{2} Q_{2}(x, y)+\ldots+k^{n+1} Q_{n+1}(x, y) \in Z(R) \text { for all } x, y \in R,
$$

where $Q_{i}(x, y)$ denotes the sum of the terms in which $y$ appears $i$ times. By Lemma 1 , we have for all $x, y \in R$,

$$
[[\delta(x), x], y]+[[\delta(x), y], x]+n[[\Delta(x, x, \ldots, y), x], x] \in Z(R) .
$$

Again replacing $y$ by $x y$ in the above expression, we get

$$
\begin{gathered}
x([[\delta(x), x], y]+[[\delta(x), y], x]+n[[\Delta(x, x, \ldots, y), x], x])+(n+2)[[\delta(x), x], x] y \\
+(2 n+1)[\delta(x), x][y, x]+n \delta(x)[[y, x], x] \in Z(R) .
\end{gathered}
$$


Combining (2.18) with the latter relation, we find that

$$
(3 n+3)[[\delta(x), x], x][y, x]+(3 n+1)[\delta(x), x][[y, x], x]+n \delta(x)[[[y, x], x], x]=0 .
$$

Further replace $y$ by $\delta(x)$ in (2.19) to get

$$
(6 n+4)[[\delta(x), x], x][\delta(x), x]=0 .
$$

On commuting with $x$, we find that

$$
(6 n+4)[[\delta(x), x], x]^{2}=0 .
$$

Next, on replacing $y$ by $[\delta(x), x]$ in (2.19) and using the given condition, we have

$$
(3 n+3)[[\delta(x), x], x]^{2}=0 .
$$

Now combine (2.20) and (2.21) to get $2[[\delta(x), x], x]^{2}=0$.

Since, $R$ is $(\mathrm{n}+1)$ !-torsion free and also the center of semi-prime ring is free from nilpotent element, we have $[[\delta(x), x], x]=0$. From Theorem $1, \delta$ is commuting on $R$.

Combining Theorem 2 with Lemma 3, we can prove the following:

Corollary 1. Let $n \geq 2$ be a fixed positive integer and $R$ be $a(n+1)$ !-torsion free semi-prime ring admitting a non-zero permuting $n$-derivation $\Delta$ such that the trace $\delta$ satisfies $[[\delta(x), x], x] \in Z(R)$ for all $x \in R$. Then $R$ is commutative.

Theorem 3. Let $n \geq 1$ be a fixed positive integer and $R$ be a non-commutative $n$ !-torsion free prime ring admitting a permuting generalized $n$-derivation $\Omega$ with associated $n$-derivation $\Delta$ such that the trace $\omega$ of $\Omega$ is commuting on $R$. Then $\Omega$ is a left $n$-multiplier on $R$.

Proof. Our hypothesis yields that

$$
[\omega(x), x]=0 \text { for all } x \in R \text {. }
$$

It can be easily seen that

$$
\omega(x+y)=\omega(x)+\omega(y)+\sum_{r=1}^{n-1}\left(\begin{array}{l}
n \\
r
\end{array}\right) p_{r}(x, y) \text { for all } x, y \in R
$$

where $p_{r}(x, y)=\Omega(\underbrace{x, x, \cdots, x}_{(n-r) \text {-times }}, \underbrace{y, y, \cdots, y}_{r \text {-times }})$.

Substituting $x+\lambda y$, where $\lambda(1 \leq \lambda \leq n)$ is a positive integer, in place of $x$ in the above equation we obtain

$$
\begin{aligned}
0 & =[\omega(x+\lambda y), x+\lambda y] \\
& =\left[\omega(x)+\omega(\lambda y)+\sum_{r=1}^{n-1}\left(\begin{array}{l}
n \\
r
\end{array}\right) p_{r}(x, \lambda y), x+\lambda y\right] .
\end{aligned}
$$


Using (2.22), we have

$$
\begin{aligned}
0= & \lambda\left\{[\omega(x), y]+\left(\begin{array}{l}
n \\
1
\end{array}\right)\left[p_{1}(x, y), x\right]\right\}+\lambda^{2}\left\{\left(\begin{array}{l}
n \\
1
\end{array}\right)\left[p_{1}(x, y), y\right]\right. \\
& \left.+\left(\begin{array}{c}
n \\
2
\end{array}\right)\left[p_{2}(x, y), x\right]\right\}+\cdots+\lambda^{n}\{[\omega(y), x] \\
& \left.+\left(\begin{array}{c}
n \\
n-1
\end{array}\right)\left[p_{n-1}(x, y), x\right]\right\} \text { for all } x, y \in R
\end{aligned}
$$

Implementing Lemma 1 we get

$$
\begin{aligned}
0 & =[\omega(x), y]+\left(\begin{array}{l}
n \\
1
\end{array}\right)\left[p_{1}(x, y), x\right] \\
& =[\omega(x), y]+n[\Omega(x, x, \cdots, y), x] .
\end{aligned}
$$

Replacing $y$ by $y x$ we obtain

$$
\begin{aligned}
0 & =y[\omega(x), x]+[\omega(x), y] x+n[\Omega(x, x, \cdots, y) x+y \Delta(x, x, \cdots, x), x] \\
& =[\omega(x), y] x+n y[\delta(x), x]+n[y, x] \delta(x)+n[\Omega(x, x, \cdots, y), x] x \\
& =n[y, x] \delta(x)+n y[\delta(x), x] .
\end{aligned}
$$

Again replacing $y$ by $z y$ for any $z \in R$ we have $[z, x] y \delta(x)=0$ for all $x, y, z \in R$. Since $R$ is prime we find that for any $x \notin Z(R), \delta(x)=0$. Now for any $y \in Z(R)$ and $x \notin Z(R), x+\lambda y \notin Z(R)$. Hence,

$$
\begin{aligned}
0 & =\delta(x+\lambda y) \\
& =\lambda p_{1}(x, y)+\cdots+\lambda^{n-1} p_{n-1}(x, y)+\lambda^{n} \delta(y) .
\end{aligned}
$$

Using Lemma 2 we obtain $\delta(y)=0$ for any $y \in Z(R)$. Thus, $\delta(x)=0$ for all $x \in R$. Lemma 1 yields that $\Delta=0$. This implies that $\Omega$ acts as a left $n$-multiplier.

Theorem 4. Let $n \geq 2$ be a fixed positive integer and $R$ be an $n$ !-torsion free semi-prime ring admitting a permuting generalized $n$-derivation $\Omega$ with associated $n$-derivation $\Delta$ such that the trace $w$ of $\Omega$ is centralizing on $R$. Then $w$ is commuting on $R$.

Proof. It is given that $[w(x), x] \in Z(R)$ for all $x \in R$. Using the similar arguments as used in Theorem 2, we obtain

$$
[w(x), y]+n[\Omega(x, x, \ldots, y), x] \in Z(R) \text { for all } x, y \in R .
$$

Replacing $y$ by $y x$, we obtain

$y[w(x), x]+[w(x), y] x+n[\Omega(x, x, \ldots, y), x] x+n y[\delta(x), x]+n[y, x] \delta(x) \in Z(R)$. 
Now in view of (2.23), we find that

$$
\begin{aligned}
0= & {[y, x][w(x), x]+n[y, x][\delta(x), x]+n y[[\delta(x), x], x] } \\
& +n[y, x][\delta(x), x]+n[[y, x], x] \delta(x) \text { for all } x, y \in R .
\end{aligned}
$$

Again replace $y$ by $w(x) y$ to get

$$
\begin{aligned}
0= & w(x)[y, x][w(x), x]+[w(x), x] y[w(x), x]+n w(x)[y, x][\delta(x), x] \\
& +n[w(x), x] y[\delta(x), x]+n w(x) y[[\delta(x), x], x]+n w(x)[y, x][\delta(x), x] \\
& +n[w(x), x] y[\delta(x), x]+n[w(x), x][y, x] \delta(x)+n w(x)[[y, x], x] \delta(x) \\
& +n[w(x), x][y, x] \delta(x)+n[[w(x), x], x] y \delta(x) .
\end{aligned}
$$

Using (2.24) and the given condition, we find that

$$
[w(x), x] y[w(x), x]+2 n[w(x), x] y[\delta(x), x]+2 n[w(x), x][y, x] \delta(x)=0 .
$$

Further, replacing $y$ by $[w(x), x]^{2}$ in (2.25) and using the given condition, we have

$$
[w(x), x]^{4}+2 n[w(x), x]^{3}[\delta(x), x]=0 \text { for all } x \in R .
$$

Again, replace $y$ by $y z$ in (2.25) and use (2.25), to get

$$
2 n[w(x), x][y, x] z \delta(x)=0 \text { for all } x, y, z \in R .
$$

Next, we replace $y$ by $w(x)$ and $z$ by $[w(x), x]$ to find that $2 n[w(x), x]^{3} \delta(x)=$ 0 for all $x \in R$. On commuting the latter relation with $x$ and using the given condition, we have

$$
2 n[w(x), x]^{3}[\delta(x), x]=0 \text { for all } x \in R .
$$

From (2.26) and (2.28), we find that $[w(x), x]^{4}=0$. Since the center of a semi-prime ring does not contain any nilpotent element, we get $[w(x), x]=0$.

Corollary 2. Let $n \geq 2$ be a fixed positive integer and $R$ be a non-commutative $n$ !-torsion free semi-prime ring admitting a permuting generalized $n$-derivation $\Omega$ with associated $n$-derivation $\Delta$ such that the trace $w$ of $\Omega$ is centralizing on $R$. Then $\Omega$ is a left $n$-multiplier on $R$.

Proof. By Theorem 3 and Theorem 2, we get the required result.

In conclusion, if we look at Theorem 2 closely, it is tempting to conjecture as follows:

Conjecture 1. Let $R$ be a semi-prime ring with suitable torsion restrictions and $\Delta$ be a non-zero permuting $n$-derivation. Suppose that for some integer $m \geq 1, \delta_{m}(x) \in$ $Z(R)$ for all $x \in R$ where $\delta_{k+1}(x)=\left[\delta_{k}(x), x\right]$ for $k>1$ and $\delta_{1}(x)=\delta(x)$ stands for the trace of $\Delta$. Then $[\delta(x), x]=0$ for all $x \in R$.

\section{ACKNOWLEDGEMENT}

The authors are indebted to the referee for his/her useful suggestions and valuable comments. 


\section{REFERENCES}

[1] M. Ashraf, "On symmetric bi-derivations in rings," Rend. Istit. Mat. Univ. Trieste, vol. 31, no. 1-2, pp. 25-36, 1999.

[2] M. Ashraf and M. R. Jamal, "Traces of Permuting $n$-additive maps and permuting $n$ - derivations of rings," Mediterr. J. Math, vol. 11, no. 2, pp. 287-297, 2014, doi: 10.1017/S00009-015-0606-3.

[3] M. Ashraf, N. Parveen, and M. R. Jamal, "Traces of permuting $n$-derivations and commutativity of rings," Southeast Asian Bulletin of Mathematics, vol. 38, pp. 321-332, 2014.

[4] M. Brešar, "On the distance of the composition of two derivations to the generalized derivations," Glasgow Math. J., vol. 33, pp. 89-93, 1991, doi: 10.1017/S0017089500008077.

[5] M. Brešar, "Commuting maps: a survey," Taiwanese J. Math., vol. 8, no. 3, pp. 361-397, 2004.

[6] Q. Deng and H. E. Bell, "On derivations and commutativity in semiprime rings." Comm. Algebra, vol. 23, no. 10, pp. 3705-3713, 1995, doi: 10.1080/00927879508825427.

[7] K. Park, "On prime and semiprime rings with symmetric $n$-derivations," J. Chungcheong Math. Soc., vol. 22, no. 3, pp. 451-458, 2009.

[8] E. Posner, "Derivations in prime rings," Proc. Amer. Math. Soc., vol. 8, pp. 1093-1100, 1957, doi: 10.1090/S0002-9939-1957-0095863-0.

[9] J. Vukman, "Symmetric bi-derivations on prime and semiprime rings," Aequationes Math., vol. 38, pp. 245-254, 1989, doi: 10.1007/BF01840009.

[10] J. Vukman, "Two results concerning symmetric bi-derivations on prime rings," Aequationes Math., vol. 40, pp. 181-189, 1990, doi: 10.1007/BF0211294.

Authors' addresses

Mohammad Ashraf

Aligarh Muslim University, Department of Mathematics Aligarh, India

E-mail address: mashraf80@hotmail.com

Almas Khan

Aligarh Muslim University, Department of Mathematics Aligarh, India

E-mail address: almasleo@gmail.com

Malik Rashid Jamal

Integral University, Department of Mathematics Lucknow, India

E-mail address: rashidmaths@gmail.com 